\title{
High-performance Dual-band Wireless Capacitive Pressure Sensor
}

\author{
M. M. Abutaleb \\ Electronics, Communication, and Computer \\ Helwan University \\ Egypt
}

\author{
A. M. Anis \\ Electronics, Communication, and Computer \\ Helwan University \\ Egypt
}

\begin{abstract}
This paper presents the design of a high-performance dualband wireless capacitive pressure sensor operating in harsh environment. The proposed design is composed of an absolute capacitive pressure sensor, pressure controlled oscillator (PCO), class-E power amplifier (PA) and an antenna. The sensor node collects and transmits the pressure variation in the term of frequency output wirelessly to a nearby base station. Simulation results show that the low frequency band can be tuned from 2.1 to $2.6 \mathrm{GHz}$ while the high frequency band ranging from 4.6 to $5.1 \mathrm{GHz}$ in a pressure range from 5 to 10 $\mathrm{MPa}$ with total output power from 16 to $14 \mathrm{dBm}$ at $0.35 \mu \mathrm{m}$ CMOS technology.
\end{abstract}

\section{Keywords}

Capacitive pressure sensor, class-E PA, LC-based oscillator, dual-band

\section{INTRODUCTION}

Micro pressure sensors suitable for operating in harsh environments are needed for applications such as automotive industry, aerospace, industrial automatic control and nuclear power stations. Most existing pressure sensors do not satisfy the needs and suffer from severe performance degradation and failure at such environments conditions. These sensors require replacement of the conventional structural, mechanical materials and readout interface circuits with other generic and excellent ones for improving performance and using with pressure sensors in smart portable and wireless microsystems applications.

Figure 1 shows a block diagram of the proposed pressure sensor, it consists of capacitive pressure sensor, pressure controlled oscillator (PCO) readout circuit, power amplifier (PA) and antenna. The capacitive pressure sensor converts the pressure information to a capacitance change; the $\mathrm{PCO}$ converts the capacitance change into output frequency variation.

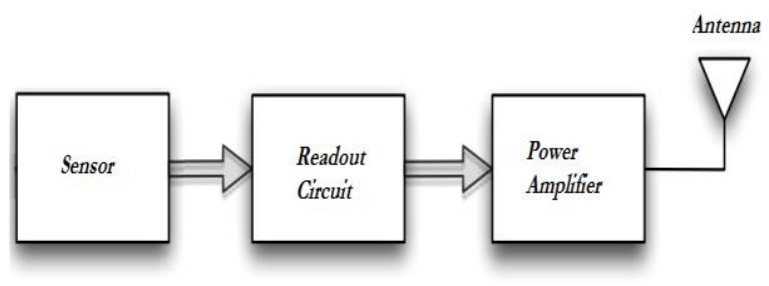

Fig 1: Block diagram of wireless sensor node

The controlled oscillators designed in CMOS technologies have become nowadays a real solution in the band of radio frequencies because of having achieved low power consumption and low phase noise values. The strong combination of very low phase noise specifications with very low power consumption pushes designers to use LCoscillators. A cross-coupled CMOS LC-oscillator provides minimum power consumption [1]. Also, the future transceiver system for wireless networks must be able to adapt itself to various applications and environments, so multi-mode is the trend [2]. In multi-mode systems, it would be ideal to use a signal oscillator which can generate multiple frequencies. Therefore, a dual-band cross-coupled CMOS LC-oscillator is proposed here. The power amplifier (PA) is the last stage before the antenna. The main function of the PA stage is to boost the power of the radio frequency signal to the required level in an efficient manner. The proposed sensor node is expected to be light weight, low power consumption, small size and low cost.

\section{CAPACITIVE PRESSURE SENSOR}

Capacitive pressure sensor measures the deflections of sensor diaphragm and total capacitance in response to pressure. Sensor diaphragm defined small-deflection if the center deflection is smaller than $20 \%$ of its thickness and defined large-deflection if the center deflection up to three times the diaphragm thickness [3]. Figure 2 shows a cross sectional view of a pressure sensor sensing cell in its two modes of operation.
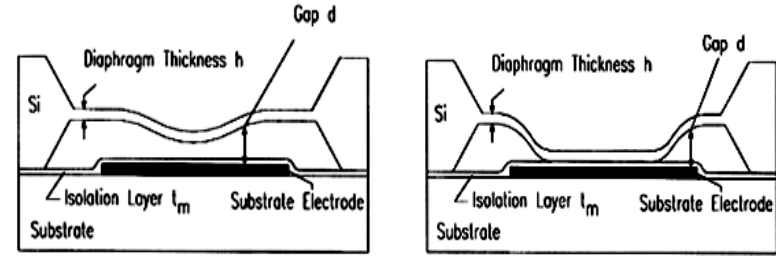

Fig 2: Cross-section view of capacitive pressure sensor (a) small-deflection and (b) large-deflection

Compared to a square- or rectangular-shaped diaphragm, circular-geometry exhibits simplicity in design, low fabrication cost, improved sensitivity and less sensitive to temperature variations for the same effective area. The capacitive pressure sensor consists of an array of circular pressure sensitive diaphragms (sensing cells), which serve as the top electrodes [4]. The diaphragms are switched in parallel to increase the value of the capacitance. Applied pressure causes the diaphragm to deflect and the capacitance to change.

\section{READOUT CIRCUIT}

PCO readout circuit converts total capacitance related to the pressure applied into output frequency variation. A crosscoupled CMOS LC-oscillator is proposed as a readout circuit, since it has very few transistors, is easy to implement, 
consumes low power, and is differential in nature which is suitable for wireless telecommunication applications. Figure 3 shows a schematic diagram of a dual- band cross-coupled CMOS LC-oscillator readout circuit. The operation principle of the cross-coupled LC-oscillator and the calculation of total negative resistance are presented in [5]. In this work, a switching transistor concept is proposed and realized in a 2.6 GHz /5.1 GHz dual-band LC-oscillator by the advanced $0.35 \mu \mathrm{m}$ CMOS technologies. Using the switching transistors, we can change the configuration of the inductor network resulting in the variation of inductance in the tank circuit. This operational mechanism provides the necessary oscillation frequency bands for various applications and environments.

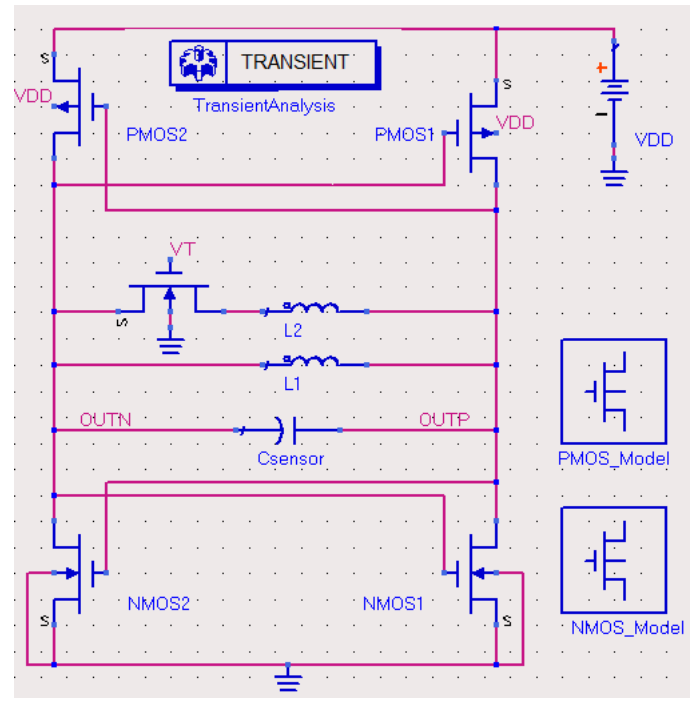

Fig 3: Schematic of a proposed readout circuit

The gate of the switching transistor is determined by the switch voltage (VT). For a high switching control voltage, the switching transistor is on, resulting in a lower in-parallel inductance consisting of the L1 and L2 as shown in Fig. 3, and it selects the high-band oscillation frequency. On the other hand, for a low switching control voltage, the switch is off, and the inductor L2 is not active. As a result, the inductance is high in the tank circuit and the LC-oscillator operates in the low-band. In general, by controlling the voltage of the switching transistors, the inductance of the tank circuit can be controlled, which generates the different oscillation frequencies. In addition, the fine-tuning of the readout circuit in each band is accomplished by using the capacitive pressure sensors.

\section{POWER AMPLIFIER}

The main function of the PA is to boost output power of pressure sensor to the required level suitable for transmission. Class-E PA is the most attractive candidate in terms of circuit simplicity and high efficiency performance. At the same time, it presents less power losses for the same frequency comparing with the others PAs. Figure 4 shows the circuit topology of class-E PA.

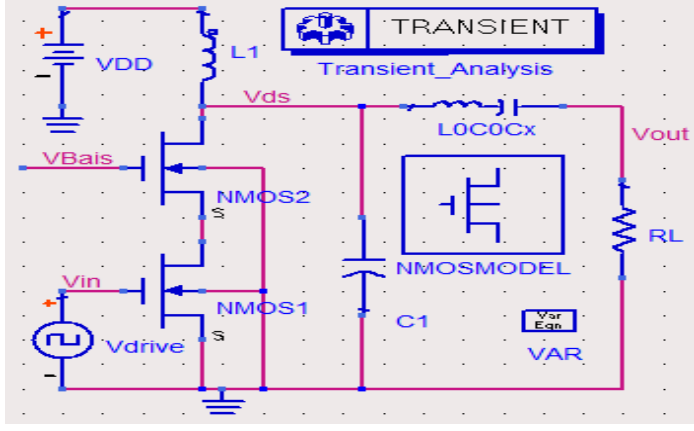

Fig 4: Schematic of cascode class-E PA

\section{SIMULATION RESULTS}

For the capacitive pressure sensor, several diaphragm materials such as Aluminum (Al) [6], Gold (Au) [7], Stainless Steel [8] and Silicon (Si) [9] are compared with SiC [4]. The maximum center deflection of the circular diaphragm, the capacitance of the pressure sensor under applying pressure loads up to $500 \mathrm{kPa}$, and maximum pressure load in normal mode of operation are summarized in table 1 .

Table 1. Diaphragm materials effect

\begin{tabular}{lccccc}
\hline \hline Symbol & \multirow{2}{*}{ Al } & Au & Si & $\begin{array}{c}\text { Stainless } \\
\text { Steel }\end{array}$ & SiC \\
\hline Deflection $(\mu \mathrm{m})$ & 0.45 & 0.41 & 0.26 & 0.24 & 0.12 \\
Capacitance $(\mathrm{pF})$ & 1.18 & 1.15 & 1.07 & 1.06 & 1.01 \\
Pressure $(\mathrm{MPa})$ & 0.7 & 0.8 & 1.2 & 1.4 & 2.7 \\
\hline \hline
\end{tabular}

It can be seen that using $\mathrm{SiC}$ material for sensor diaphragm, can reduce the deflection of sensor diaphragm and minimize the capacitance of pressure sensor that result in increasing the applied pressure range. Also, several dielectric materials such as Silicon Oxide $\left(\mathrm{SiO}_{2}\right)$, Silicon Nitride $\left(\mathrm{Si}_{3} \mathrm{~N}_{4}\right)$, Zirconium Oxide $\left(\mathrm{ZrO}_{2}\right)$ and Lanthanum Oxide $\left(\mathrm{La}_{2} \mathrm{O}_{3}\right)$ are compared with Hafnium Oxide $\mathrm{HfO}_{2}$. The dielectric capacitance and the overall capacitance of the pressure sensor at no pressure load are summarized in table 2. Also, it can be seen that increasing dielectric constant can increase dielectric capacitance that result in an increasing in the overall sensor capacitance. To provide the same sensor capacitance; the number of sensing cells can be reduced. The proposed number of sensing cells and the corresponding over all sensor capacitance at no pressure load are summarized in table 3 .

Table 2. Dielectric materials effect

\begin{tabular}{lccccc}
\hline \hline Symbol & $\mathbf{S i O}_{2}$ & $\mathbf{S i}_{3} \mathbf{N}_{\mathbf{4}}$ & $\mathbf{H f O}_{2}$ & $\mathbf{Z r O}_{\mathbf{2}}$ & $\mathbf{L a}_{2} \mathbf{O}_{\mathbf{3}}$ \\
\hline Dielectric Constant & 4 & 7.8 & 20 & 23 & 27 \\
Dielectric C $(\mathrm{pF})$ & 0.28 & 0.54 & 1.39 & 1.59 & 1.87 \\
Sensor C $(\mathrm{pF})$ & 0.98 & 1.2 & 1.5 & 1.53 & 1.56 \\
\hline \hline
\end{tabular}

Table 3. Effect of reducing number of sensing cells

\begin{tabular}{lccccc}
\hline \hline Symbol & $\mathbf{S i O}_{2}$ & $\mathbf{S i}_{3} \mathbf{N}_{\mathbf{4}}$ & $\mathbf{H f O}_{2}$ & $\mathbf{Z r O}_{2}$ & $\mathbf{L a}_{2} \mathbf{O}_{3}$ \\
\hline No. of Cells & 16 & 12 & 9 & 9 & 9 \\
Sensor C $(\mathrm{pF})$ & 0.98 & 0.93 & 0.85 & 0.87 & 0.89 \\
\hline \hline
\end{tabular}

It can be seen that using $\mathrm{HfO}_{2}$ as an insulator can reduce the number of sensing cells and reduce total area. Finally, a comparison between $\mathrm{SiC} / \mathrm{HfO}_{2}$-based pressure sensor and 
$\mathrm{SiC} / \mathrm{SiO}_{2}$-based pressure sensor at no pressure load is given in table 4.

Table 4. Effect of the proposed sensor materials

\begin{tabular}{lcc}
\hline \hline Symbol & $\begin{array}{c}\text { SiC/HfO } \\
\text { Diaphragm }\end{array}$ & $\begin{array}{c}\text { SiC/SiO } \\
\text { Diaphragm }\end{array}$ \\
\hline Average Dielectric Constant & 15 & 7 \\
No. of Sensing Cells N & 9 & 16 \\
Sensor Capacitance (pF) & 0.85 & 0.98 \\
Touch Point Pressure (MPa) & 4.3 & 2.7 \\
\hline \hline
\end{tabular}

Figure 5 shows the radial distance versus diaphragm deflection under different pressure loads. The contact area gradually expands as the pressure loading increase. Figure 6 shows the total capacitance of the sensor with applying different pressure loads. The capacitance is almost linearly at a certain range beyond the touch point load. The simulated results show that the capacitance of the pressure sensor changes from 1.6 to $2.2 \mathrm{pF}$ when pressure increased the linear range from 5 to $10 \mathrm{MPa}$.

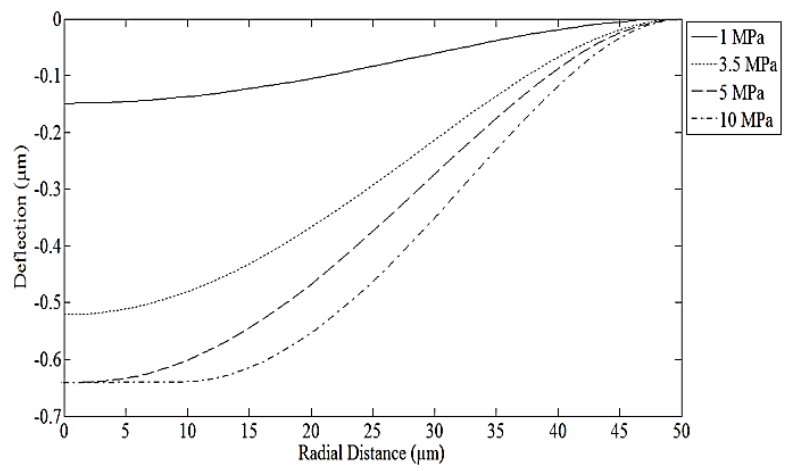

Fig 5: Diaphragm deflections versus pressure loads up to $10 \mathrm{MPa}$

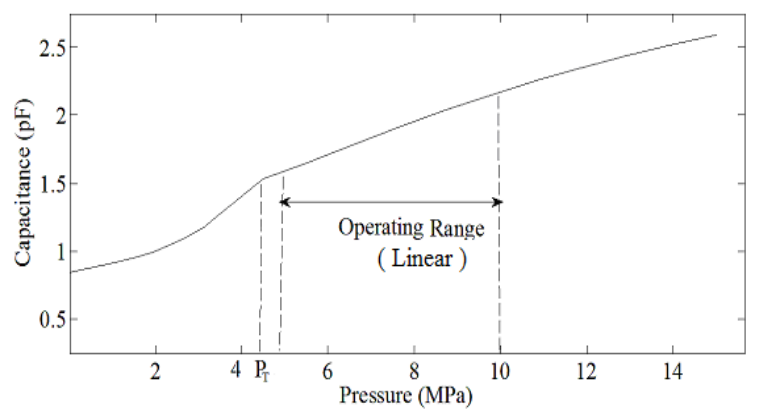

Fig 6: Total capacitance of pressure sensor versus different pressure loads

The $\operatorname{ADS}^{\circledR}$ simulator is used to simulate the proposed PCO readout circuit for the dual-band operation. The performance is simulated at a 3.3 supply voltage. As the circuit operated at the low-band and by changing the sensor capacitance from 1.6 to $2.2 \mathrm{pF}$ due to applied pressure from 5 to $10 \mathrm{MPa}$, shown in Fig. 7(a), the oscillation frequencies can be tuned between 2.1 and $2.6 \mathrm{GHz}$. As the results shown in Fig. 7(b), the tuning range for the high-band is between 4.6 and $5.1 \mathrm{GHz}$.
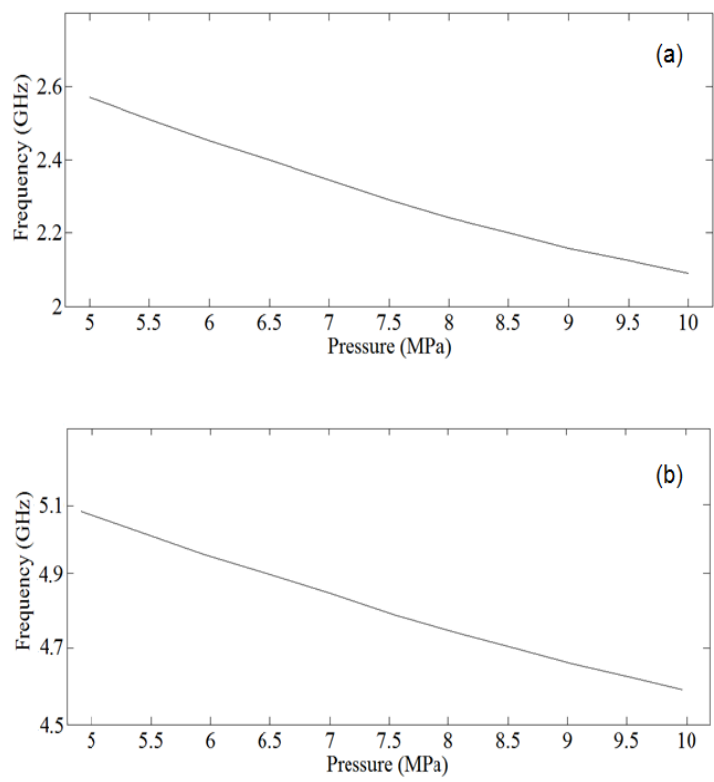

Fig 7: Oscillation frequency versus pressure at low-band (a), and at high-band (b)

Again, the $\mathrm{ADS}^{\circledR}$ simulator is used to simulate the class-E PA circuit. The drain-to-source voltage with different values of bias voltage is illustrated in Fig. 8. Figure 9 and 10 show the frequency response of the output voltage and the output power of the proposed pressure sensor node. The simulated results show that when the output power varied from 14 to $16 \mathrm{dBm}$ and the overall efficiency changed from $34 \%$ to $52 \%$ in the pressure range from 5 to $10 \mathrm{MPa}$.

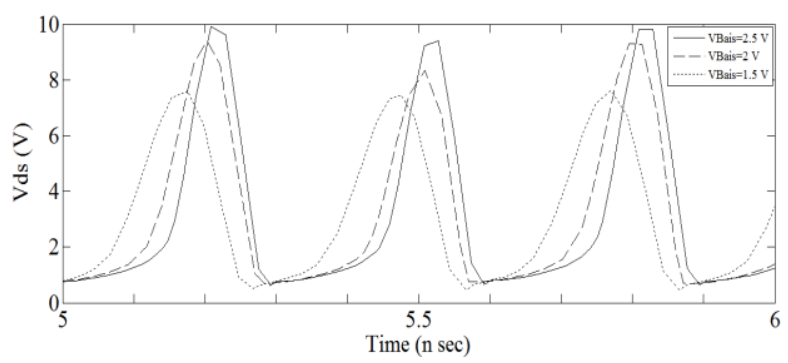

Fig 8: The drain-to-source voltage of cascode class-E PA

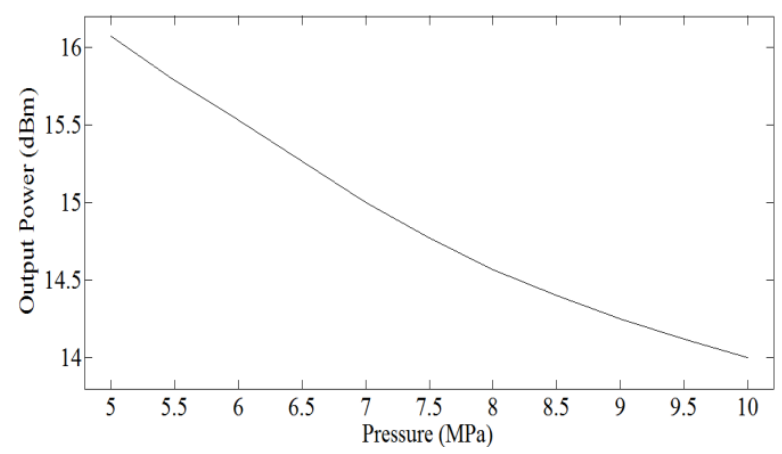

Fig 9: Output power with pressure

\section{CONCLUSION}

In this paper, a dual-band wireless pressure sensor for high pressure and various frequency applications has been 
presented by the $0.35 \mu \mathrm{m}$ CMOS technology. For the pressure capacitive sensor, $\mathrm{SiC}$ diaphragm material has been selected to increase the applied pressure range and $\mathrm{HfO}_{2}$ insulator has been selected in order to reduce sensor area. The proposed pressure-controlled oscillator includes sensor capacitance array, on-chip inductors and switching transistors, which achieves a dual-band operation and a lower phase noise with lower power consumption. The proposed sensor node gives an output with low frequency range from 2.6 to $2.1 \mathrm{GHz}$ and high frequency range from 5.1 to $4.6 \mathrm{GHz}$ in the pressure range from 5 to $10 \mathrm{MPa}$ with total output power from 16 to 14 $\mathrm{dBm}$. The frequency and power sensitivity with respect to the applied pressure loads are $0.08 \mathrm{GHz} / \mathrm{MPa}$ and $0.4 \mathrm{dBm} / \mathrm{MPa}$ respectively.

\section{REFERENCES}

[1] M.A. Nandanwar, M.A. Gaikwad and D. Dandekar, "Low Power Low Phase Noise CMOS LC VCO-A Review," International Journal of Applied Information Systems, vol. 3, no. 3, pp. 37-41, July 2012.

[2] Bo Zhao, "An Ultra-Low-Supply Dual-Band VCO for wireless Sensor Networks, "International Conference on Communication, Circuits and Systems, pp. 797-801, July 2009.

[3] S. Timoshenko and S. Woinosky-Krieger, "Theory of Plates and Shells," New York, McGraw-Hill., 1987.

[4] A.M. Anis, M.M. Abutaleb, H.F. Ragai, M.I. Eladawy, "SiC Capacitive pressure Sensor Node for Harsh
Industrial Environment," International Conference on Computational Intelligence, Modelling \& Simulation, pp. 413 - 416, Sept. 2011.

[5] A.M. Anis, M.M. Abutaleb, H.F. Ragai, M.I. Eladawy, "Design of SiC Capacitive Pressure Sensor with LCbased Oscillator Readout Circuit," World Academy of Science, Engineering and Technology, issue 64, pp. 699 702, April 2012.

[6] Dai C. L., Lu P. W., Chang C., Liu C. Y., "Capacitive Micro Pressure Sensor Integrated with a Ring Oscillator Circuit on Chip," Sensors 9, no. 12, pp. 10158-10170, 2009.

[7] P. Eswaran and S. Malarvizhi, "Design Analysis of MEMS Capacitive Differential Pressure Sensor for Aircraft Altimeter," International Journal of Applied Physics and Mathematics, vol. 2, no. 1, Jan. 2012.

[8] S. P. Chang, M. G. Allen, "Capacitive Pressure Sensors with Stainless Steel Diaphragm and Substrate," Journal of Micromechanics and Microengineering, vol. 14, no. 4, pp. 612-618, April 2004.

[9] M. Suster, W. H. Ko, D. J. Young, “An Optically Powered Wireless Telemetry Module for HighTemperature MEMS Sensing and Communication," IEEE Journal of Microelectromechanical Systems, pp. 536-541, June 2004. 